Andriy Bandura*, Oleh Skaskiv, and Liana Smolovyk

\title{
Slice holomorphic solutions of some directional differential equations with bounded $L$-index in the same direction
}

https://doi.org/10.1515/dema-2019-0043

Received July 27, 2019; accepted November 3, 2019

Abstract: In the paper we investigate slice holomorphic functions $F: \mathbb{C}^{n} \rightarrow \mathbb{C}$ having bounded $L$-index in a direction, i.e. these functions are entire on every slice $\left\{z^{0}+t \mathbf{b}: t \in \mathbb{C}\right\}$ for an arbitrary $z^{0} \in \mathbb{C}^{n}$ and for the fixed direction $\mathbf{b} \in \mathbb{C}^{n} \backslash\{\mathbf{0}\}$, and $\left(\exists m_{0} \in \mathbb{Z}_{+}\right)\left(\forall m \in \mathbb{Z}_{+}\right)\left(\forall z \in \mathbb{C}^{n}\right)$ the following inequality holds

$$
\frac{\left|\partial_{\mathbf{b}}^{m} F(z)\right|}{m ! L^{m}(z)} \leq \max _{0 \leq k \leq m_{0}} \frac{\left|\partial_{\mathbf{b}}^{k} F(z)\right|}{k ! L^{k}(z)},
$$

where $\mathbf{L}: \mathbb{C}^{n} \rightarrow \mathbb{R}_{+}$is a positive continuous function, $\partial_{\mathbf{b}} F(z)=\left.\frac{d}{d t} F(z+t \mathbf{b})\right|_{t=0}, \partial_{\mathbf{b}}^{p} F=\partial_{\mathbf{b}}\left(\partial_{\mathbf{b}}^{p-1} F\right)$ for $p \geq 2$. Also, we consider index boundedness in the direction of slice holomorphic solutions of some partial differential equations with partial derivatives in the same direction. There are established sufficient conditions providing the boundedness of $L$-index in the same direction for every slie holomorphic solutions of these equations.

Keywords: bounded index, bounded $L$-index in direction, slice function, holomorphic function, bounded $l$-index, directional differential equation

MSC: 32A10, 32A17, 32A37, 30H99, 30A05

\section{Introduction}

Here we continue our investigations initialized in $[1,2]$. There was introduced a concept of $L$-index boundedness in direction for slice entire functions of several complex variables and obtained many criteria of $L$-index boundedness in direction. Here we present some applications of these criteria to deduce sufficient conditions providing that sum, product of slice entire functions is a function of bounded $L$-index in direction. Also we consider slice entire solutions of directional differential equations. Since functions of bounded index has many applications in analytic theory of differential equations [3-5] we study local behavior of slice holomorphic functions of bounded $L$-index in direction which satisfy some linear higher-order directional differential equations.

In conclusions from [1] there was posed a question on the additional conditions, providing index boundedness of every slice holomorphic solutions for linear higher-order partial differential equations with slice holomorphic coefficients. So, the topic is central in this investigation.

We also consider a more general problem.

\footnotetext{
^Corresponding Author: Andriy Bandura: Department of Advanced Mathematics, Ivano-Frankivsk National Technical University of Oil and Gas, 15 Karpatska Street, Ivano-Frankivsk, 76019, Ukraine; E-mail: andriykopanytsia@gmail.com Oleh Skaskiv: Department of Mathematics and Mechanics, Ivan Franko National University of L'viv, 1 Universytetska Street, Lviv, 79000, Ukraine; E-mail: olskask@gmail.com

Liana Smolovyk: Department of Advanced Mathematics, Ivano-Frankivsk National Technical University of Oil and Gas, 15 Karpatska Street, Ivano-Frankivsk, 76019, Ukraine; E-mail: lil02smo@gmail.com
} 
Problem 1. [2] Is it possible to deduce main facts of theory of entire functions having bounded L-index in the direction $\mathbf{b} \in \mathbb{C}^{n} \backslash\{0\}$ for functions which are holomorphic on the slices $\left\{z^{0}+t \mathbf{b}: t \in \mathbb{C}\right\}$ and are joint continuous?

Let us introduce some notations from $[1,2]$. Let $\mathbb{R}_{+}=(0,+\infty), \mathbb{R}_{+}^{*}=[0,+\infty), \mathbf{0}=(0, \ldots, 0), \mathbf{b}=\left(b_{1}, \ldots, b_{n}\right) \in$ $\mathbb{C}^{n} \backslash\{\boldsymbol{0}\}$ be a given direciton, $L: \mathbb{C}^{n} \rightarrow \mathbb{R}_{+}$be a continuous function, $F: \mathbb{C}^{n} \rightarrow \mathbb{C}$ an entire function. The slice functions on a line $\left\{z^{0}+t \mathbf{b}: t \in \mathbb{C}\right\}$ for fixed $z^{0} \in \mathbb{C}^{n}$ we will denote as $g_{z^{0}}(t)=F\left(z^{0}+t \mathbf{b}\right)$ and $l_{z^{0}}(t)=L\left(z^{0}+t \mathbf{b}\right)$. Besides, we denote by $\langle a, c\rangle=\sum_{j=1}^{n} a_{j} \overline{c_{j}}$ the Hermitian scalar product in $\mathbb{C}^{n}$, where $a, c \in \mathbb{C}^{n}$.

Let $\widetilde{\mathcal{H}}_{\mathbf{b}}^{n}$ be a class of functions which are holomorphic on every slices $\left\{z^{0}+t \mathbf{b}: t \in \mathbb{C}\right\}$ for each $z^{0} \in \mathbb{C}^{n}$ and let $\mathcal{H}_{\mathbf{b}}^{n}$ be a class of functions from $\widetilde{\mathcal{H}}_{\mathbf{b}}^{n}$ which are joint continuous. The notation $\partial_{\mathbf{b}} F(z)$ stands for the derivative of the function $g_{z}(t)$ at the point 0 , i.e. for every $p \in \mathbb{N} \quad \partial_{\mathbf{b}}^{p} F(z)=g_{z}^{(p)}(0)$, where $g_{z}(t)=F(z+t \mathbf{b})$ is entire function of complex variable $t \in \mathbb{C}$ for given $z \in \mathbb{C}^{n}$. It is easy to check that for any $p \in \mathbb{N}$ the derivative $\partial_{\mathbf{b}}^{p} F$ is also joint continuous.

In this research, we will often call this derivative as directional derivative because if $F$ is entire function in $\mathbb{C}^{n}$ then the derivatives of the function $g_{z}(t)$ matches with directional derivatives of the function $F$. Together the hypothesis on joint continuity and the hypothesis on holomorphy in one direction do not imply holomorphy in whole $n$-dimensional complex space.

A function $F \in \mathcal{H}_{\mathbf{b}}^{n}$ is said [1] to be of bounded L-index in the direction $\mathbf{b}$, if there exists $m_{0} \in \mathbb{Z}_{+}$such that for all $m \in \mathbb{Z}_{+}$and each $z \in \mathbb{C}^{n}$ inequality

$$
\frac{\left|\partial_{\mathbf{b}}^{m} F(z)\right|}{m ! L^{m}(z)} \leq \max _{0 \leq k \leq m_{0}} \frac{\left|\partial_{\mathbf{b}}^{k} F(z)\right|}{k ! L^{k}(z)},
$$

is true. The least integer number $m_{0}$, obeying (1), is called the $L$-index in the direction $\mathbf{b}$ of the function $F$ and is denoted by $N_{\mathbf{b}}(F, L)$. If such $m_{0}$ does not exist, then we put $N_{\mathbf{b}}(F, L)=\infty$, and the function $F$ is called of unbounded $L$-index in the direction $\mathbf{b}$ in this case. If $L(z) \equiv 1$, then the function $F$ is said to be of bounded index in the direction $\mathbf{b}$ and $N_{\mathbf{b}}(F)=N_{\mathbf{b}}(F, 1)$ is called the index in the direction $\mathbf{b}$. For $n=1, \mathbf{b}=1$, $L(z)=l(z), z \in \mathbb{C}$ inequality (1) defines a function of bounded $l$-index with the $l$-index $N(F, l) \equiv N_{1}(F, l)[6,7]$, and if in addition $l(z) \equiv 1$, then we obtain a definition of index boundedness with index $N(F) \equiv N_{1}(F, 1)$ $[8,9]$. It is also worth to mention paper [10], which introduces the concept of generalized index. It is quite close to the bounded $l$-index. Let $N_{\mathbf{b}}\left(F, L, z^{0}\right)$ stands for the $L$-index in the direction $\mathbf{b}$ of the function $F$ at the point $z^{0}$, i.e., it is the least integer $m_{0}$, for which inequality (1) is satisfied at this point $z=z^{0}$. By analogy, the notation $N\left(f, l, z^{0}\right)$ is defined if $n=1$, i.e. in the case of functions of one variable.

Note that the positivity and continuity of the function $L$ are weak restrictions to deduce constructive results. Thus, we assume additional restrictions by the function $L$.

Let us denote

$$
\lambda_{\mathbf{b}}(\eta)=\sup _{z \in \mathbb{C}^{n}} \sup _{t_{1}, t_{2} \in \mathbb{C}}\left\{\frac{L\left(z+t_{1} \mathbf{b}\right)}{L\left(z+t_{2} \mathbf{b}\right)}:\left|t_{1}-t_{2}\right| \leq \frac{\eta}{\min \left\{L\left(z+t_{1} \mathbf{b}\right), L\left(z+t_{2} \mathbf{b}\right)\right\}}\right\} .
$$

By $Q_{\mathbf{b}}^{n}$ we denote a class of positive continuous function $L: \mathbb{C}^{n} \rightarrow \mathbb{R}_{+}$, satisfying the condition

$$
(\forall \eta \geq 0): \lambda_{\mathbf{b}}(\eta)<+\infty .
$$

Moreover, it is sufficient to require validity of (2) for one value $\eta>0$.

For a positive continuous function $l(t), t \in \mathbb{C}$, and $\eta>0$ we define $\lambda(\eta) \equiv \lambda_{1}^{\mathbf{b}}(\eta)$ in the cases when $\mathbf{b}=1$, $n=1, L \equiv l$. As in [11], let $Q \equiv Q_{1}^{1}$ be a class of positive continuous functions $l(t), t \in \mathbb{C}$, obeying the condition $0<\lambda(\eta)<+\infty$ for all $\eta>0$. 


\section{Auxiliary propositions}

Proposition 1. [2] Let $L \in Q_{\mathbf{b}}^{n}, F \in \widetilde{\mathcal{H}}_{\mathbf{b}}^{n}$. If there exist $r_{1}$ and $r_{2}, 0<r_{1}<r_{2}<+\infty$, and $P_{1} \geq 1$ such that for all $z^{0} \in \mathbb{C}^{n}$ inequality

$$
\max \left\{\left|F\left(z^{0}+t \mathbf{b}\right)\right|:|t|=\frac{r_{2}}{L\left(z^{0}\right)}\right\} \leq P_{1} \max \left\{\left|F\left(z^{0}+t \mathbf{b}\right)\right|:|t|=\frac{r_{1}}{L\left(z_{0}\right)}\right\}
$$

holds, then the function $F$ has bounded L-index in the direction $\mathbf{b}$.

Theorem 1. [2] Let $L \in Q_{\mathbf{b}}^{n}$. If the function $F \in \widetilde{\mathcal{H}}_{\mathbf{b}}^{n}$ is of bounded L-index in the direction $\mathbf{b}$ then for each $R>0$ there exist $P_{2}(R) \geq 1$ and $\eta(R) \in(0, R)$ such that for every $z^{0} \in \mathbb{C}^{n}$ and some $r=r\left(z^{0}\right) \in[\eta(R), R]$ the inequality holds

$$
\max \left\{\left|F\left(z^{0}+t \mathbf{b}\right)\right|:|t|=r / L\left(z^{0}\right)\right\} \leq P_{2} \min \left\{\left|F\left(z^{0}+t \mathbf{b}\right)\right|:|t|=r / L\left(z^{0}\right)\right\} .
$$

Denote

$$
G_{r}(F):=G_{r}^{\mathbf{b}}(F):=\bigcup_{z: F(z)=0}\{z+t \mathbf{b}:|t|<r / L(z)\} .
$$

By $n_{z^{0}}(r, F)=n_{\mathbf{b}}\left(r, z^{0}, 1 / F\right):=\sum_{\left|a_{k}^{0}\right| \leq r} 1$ we denote counting function of zeros $a_{k}^{0}$ for the slice function $F\left(z^{0}+t \mathbf{b}\right)$ in the disc $\{t \in \mathbb{C}:|t| \leq r\}$. If for given $z^{0} \in \mathbb{C}^{n}$ and for all $t \in \mathbb{C} F\left(z^{0}+t \mathbf{b}\right) \equiv 0$, then we put $n_{z^{0}}(r)=-1$. Denote $n(r)=\sup _{z \in \mathbb{C}^{n}} n_{z}(r / L(z))$.

Theorem 2. [2] Let $F \in \widetilde{\mathcal{H}}_{\mathbf{b}}^{n}, L \in Q_{\mathbf{b}}^{n}$. If the function $F$ has bounded $L$-index in the direction $\mathbf{b}$, then

1) for each $r>0$ there exists $P=P(r)>0$ such that for every $z \in \mathbb{C}^{n} \backslash G_{r}^{\mathbf{b}}(F)$

$$
\left|\frac{\partial_{\mathbf{b}} F(z)}{F(z)}\right| \leq P L(z)
$$

2) for any $r>0$ there exists $\widetilde{n}(r) \in \mathbb{Z}_{+}$such that for all $z^{0} \in \mathbb{C}^{n}$ such that $F\left(z^{0}+\right.$ tb $) \equiv 0$ one has

$$
n_{\mathbf{b}}\left(\frac{r}{L\left(z^{0}\right)}, z^{0}, \frac{1}{F}\right) \leq \widetilde{n}(r) .
$$

From the proof of Theorem 2 in [2] it follows the following lemma:

Lemma 1. If a function $F \in \widetilde{\mathcal{H}}_{\mathbf{b}}^{n}$ is of bounded L-index in the direction $\mathbf{b}$, then for each $z^{0} \in \mathbb{C}^{n} \backslash G_{r}^{\mathbf{b}}(F)(r>0)$ and for every $\widetilde{a}^{k}=z^{0}+a_{k}^{0} \mathbf{b}$ such that $F\left(\widetilde{a}^{k}\right)=0$ one has

$$
\left|z^{0}-\widetilde{a}_{k}\right|>\frac{r|\mathbf{b}|}{2 L\left(z^{0}\right) \lambda_{\mathbf{b}}(r)} .
$$

Theorem 3. [2] Let $L \in Q_{\mathbf{b}}^{n}$. A function $F \in \widetilde{\mathcal{H}}_{\mathbf{b}}^{n}$ is of bounded $L$-index in the direction $\mathbf{b}$ if and only if there exist $p \in \mathbb{Z}_{+}$and $C>0$ such that for every $z \in \mathbb{C}^{n}$ one has

$$
\frac{\left|\partial_{\mathbf{b}}^{p+1} F(z)\right|}{L^{p+1}(z)} \leq C \max \left\{\frac{\left|\partial_{\mathbf{b}}^{k} F(z)\right|}{L^{k}(z)}: 0 \leq k \leq p\right\} .
$$

\section{Boundedness of $L$-index in direction of entire solutions of some linear partial differential equations}

Let us consider the following directional differential equation

$$
g_{0}(z) \partial_{\mathbf{b}}^{p} w+g_{1}(z) \partial_{\mathbf{b}}^{p-1} w+\ldots+g_{p}(z) w=h(z),
$$


where $g_{j}, h$ are functions from the class $\mathcal{H}_{\mathbf{b}}^{n}, j \in\{0,1, \ldots, p\}$. For entire functions of bounded $L$-index in direction the equation was investigated in $[12,13]$. Here we will consider the weaker assumption that the coefficients of equation (9) are slice entire functions, i.e. they are functions from the class $\widetilde{\mathcal{H}}_{\mathbf{b}}^{n}$.

We need the following proposition. Its proof is based on the proof of its analog for entire functions [14].

Lemma 2. Let $F \in \widetilde{\mathcal{H}}_{\mathbf{b}}^{n}$ be a function of bounded $L$-index in the direction $\mathbf{b}, L \in Q_{\mathbf{b}}^{n}$. Then for every $r>0$ and for every $m \in \mathbb{N}$ there exists $P=P(r, m)>1$ such that for all $z \in \mathbb{C}^{n} \backslash G_{r}^{\mathbf{b}}(F)$ the inequality $\left|\partial_{\mathbf{b}}^{m} F(z)\right| \leq P L^{m}(z)|F(z)|$ holds.

Proof. We apply Theorem 1 with $R=\frac{r}{2 \lambda_{\mathrm{b}}(r)}$. Then there exist $P_{2}=P_{2}(R) \geq 1$ and $\eta=\eta(R) \in(0, R)$ such that for all $z^{0} \in \mathbb{C}^{n}$ and some $r^{\star}=r^{\star}\left(z^{0}\right) \in[\eta(R), R]$ inequality (4) holds with $r^{\star}$ instead of $r$. Hence, by Cauchy's inequality we obtain

$$
\begin{aligned}
\frac{1}{m !}\left|\partial_{\mathbf{b}}^{m} F\left(z^{0}\right)\right| & \leq\left(\frac{L\left(z^{0}\right)}{r^{\star}}\right)^{m} \max \left\{\left|F\left(z^{0}+t \mathbf{b}\right)\right|:|t|=\frac{r^{\star}}{L\left(z^{0}\right)}\right\} \\
& \leq P_{2}\left(\frac{L\left(z^{0}\right)}{\eta}\right)^{m} \min \left\{\left|F\left(z^{0}+t \mathbf{b}\right)\right|:|t|=\frac{r^{\star}}{L\left(z^{0}\right)}\right\} .
\end{aligned}
$$

But by (7) for every $z^{0} \in \mathbb{C}^{n} \backslash G_{r}^{\mathbf{b}}(F)$ the set

$$
\left\{z^{0}+t \mathbf{b}:|t| \leq \frac{r}{2 \lambda_{2}^{\mathbf{b}}(r) L\left(z^{0}\right)}\right\}
$$

does not contain zeros of function $F\left(z^{0}+t \mathbf{b}\right)$. Therefore, applying to $\frac{1}{F\left(z^{0}+t \mathbf{b}\right)}$ the maximum modulus principle in variable $t \in \mathbb{C}$, we have $\left|F\left(z^{0}\right)\right| \geq \min \left\{\left|F\left(z^{0}+t \mathbf{b}\right)\right|:|t|=\frac{r^{*}}{L\left(z^{0}\right)}\right\}$. Thus,

$$
\left|\partial_{\mathbf{b}}^{m} F\left(z^{0}\right)\right| \leq m ! \frac{P_{2}}{\eta^{m}} L^{m}\left(z^{0}\right)\left|F\left(z^{0}\right)\right| \text {. }
$$

Hence, in view of arbitrariness of $z^{0}$, we obtain the desired inequality with $P=P_{2} m ! \eta^{-m}$.

Denote $g^{\star}(z)=h(z) \cdot \prod_{j=0}^{p} g_{j}(z), n\left(r, g^{\star}\right)=\sup _{z \in \mathbb{C}^{n}} n_{\mathbf{b}}\left(r / L(z), z, 1 / g^{\star}\right)$,

$$
r^{\star}=\sup _{s \geq 1} \frac{(s-1) \lambda_{1}(s)}{8\left(n\left(s, g^{\star}\right)+1\right)}, H(F):=\bigcup_{\substack{z \in Z_{F} \\ \forall t \in \mathbb{C} F(z+t \mathbf{b}) \equiv 0}}\{z+t \mathbf{b}: t \in \mathbb{C}\},
$$

where $Z_{F}$ is zero set of the function $F$. Using Lemma 2, we prove the following theorem. It was firstly obtained for entire functions of bounded $L$-index in direction [13].

Denote $G_{r}=\left(G_{r}(h) \backslash H(h)\right) \cup G_{r}\left(g_{0}\right) \cup \bigcup_{j=1}^{p}\left(G_{r}\left(g_{j}\right) \backslash H\left(g_{j}\right)\right)$.

Theorem 4. Let $L \in Q_{\mathbf{b}}^{n}$, and $g_{0}(z), \ldots, g_{p}(z), h(z) \in \mathcal{H}_{\mathbf{b}}^{n}$ be functions of bounded L-index in the direction $\mathbf{b}$. Suppose that there exist $r \in\left(0 ; r^{\star}\right)$ and $T>0$ such that $G_{r}$ is an unbounded set and for every $z \in \mathbb{C}^{n} \backslash G_{r}\left(g_{0}\right)$ and $j=1, \ldots, p$

$$
\left|g_{j}(z)\right| \leq T L^{j}(z)\left|g_{0}(z)\right| \text {. }
$$

Then every function $F \in \mathcal{H}_{\mathbf{b}}^{n}$ such that (9) is valid, has bounded L-index in the direction $\mathbf{b}$.

Proof. One should observe that the condition $F \in \mathcal{H}_{\mathbf{b}}^{n}$ implies $\partial_{\mathbf{b}}^{m} F \in \mathcal{H}_{\mathbf{b}}^{n}$ for all $m \in \mathbb{N}$. Besides, Theorem 2 and restrictions of Theorem 4 provides validity of inequalities $n\left(r, g^{\star}\right)<+\infty$ and $r^{\star}>0$.

From conditions of Theorem it follows that $\mathbb{C}^{n} \backslash G_{r} \neq \emptyset$. Lemma 2 and inequality (10) yield that there exist $r \in\left(0 ; r^{\star}\right]$ and $T^{\star}>0$ such that for all $z \in \mathbb{C}^{n} \backslash G_{r}$

$$
\begin{aligned}
\left|\partial_{\mathbf{b}} h(z)\right| \leq T^{\star}|h(z)| L(z), \quad\left|g_{j}(z)\right| \leq T^{\star}\left|g_{0}(z)\right| L^{j}(z), j \in\{1,2, \ldots, p,\}, \\
\left|\partial_{\mathbf{b}} g_{j}(z)\right| \leq P(r) L(z)\left|g_{j}(z)\right| \leq T^{\star}(r)\left|g_{0}(z)\right| L^{j+1}(z), j \in\{0,1,2, \ldots, p,\} .
\end{aligned}
$$


Evaluate the derivative in the direction b in Equation (9)

$$
g_{0}(z) \partial_{\mathbf{b}}^{p+1} F(z)+\sum_{j=1}^{p} g_{j}(z) \partial_{\mathbf{b}}^{p+1-i} F(z)+\sum_{j=0}^{p} \partial_{\mathbf{b}} g_{j}(z) \partial_{\mathbf{b}}^{p-i} F(z)=\partial_{\mathbf{b}} h(z)
$$

The obtained equality means that for all $z \in \mathbb{C}^{n} \backslash G_{r}$ :

$$
\begin{aligned}
\left|g_{0}(z)\right|\left|\partial_{\mathbf{b}}^{p+1} F(z)\right| & \leq\left|\partial_{\mathbf{b}} h(z)\right|+\sum_{j=1}^{p}\left|g_{j}(z)\right|\left|\partial_{\mathbf{b}}^{p+1-j} F(z)\right|+\sum_{j=0}^{p}\left|\partial_{\mathbf{b}} g_{j}(z)\right|\left|\partial_{\mathbf{b}}^{p-j} F(z)\right| \\
& \leq T^{\star}|h(z)| L(z)+\sum_{j=1}^{p}\left|g_{j}(z)\right|\left|\partial_{\mathbf{b}}^{p+1-j} F(z)\right|+\sum_{j=0}^{p}\left|\partial_{\mathbf{b}} g_{j}(z)\right|\left|\partial_{\mathbf{b}}^{p-j} F(z)\right| \\
& \leq T^{\star} L(z) \sum_{j=0}^{p}\left|g_{j}(z)\right|\left|\partial_{\mathbf{b}}^{p-j} F(z)\right|+\sum_{j=1}^{p}\left|g_{j}(z)\right|\left|\partial_{\mathbf{b}}^{p+1-j} F(z)\right|+\sum_{j=0}^{p}\left|\partial_{\mathbf{b}} g_{j}(z)\right|\left|\partial_{\mathbf{b}}^{p-j} F(z)\right| \\
& \leq T^{\star}\left|g_{0}(z)\right|\left(T^{\star} L(z) \sum_{j=0}^{p} L^{j}(z)\left|\partial_{\mathbf{b}}^{p-j} F(z)\right|+\sum_{j=1}^{p} L^{j}(z)\left|\partial_{\mathbf{b}}^{p+1-i} F(z)\right|+\sum_{j=0}^{p} L^{j+1}(z)\left|\partial_{\mathbf{b}}^{p-j} F(z)\right|\right) \\
& \left.=T^{\star}\left|g_{0}(z)\right| L^{p+1}(z)\left|\left(T^{\star}+1\right) \times \sum_{j=0}^{p} \frac{1}{L^{p-j}(z)}\right| \partial_{\mathbf{b}}^{p-j} F(z)\left|+\sum_{j=1}^{p} \frac{1}{L^{p+1-j}(z)}\right| \partial_{\mathbf{b}}^{p+1-i} F(z) \mid\right) \\
& \leq T^{\star}\left(\left(T^{\star}+1\right)(p+1)+p\right)\left|g_{0}(z)\right| L^{p+1}(z) \max _{0 \leq j \leq p} \frac{\left|\partial_{\mathbf{b}}^{j} F(z)\right|}{L^{j}(z)} .
\end{aligned}
$$

Thus, there exists $P_{3}>0$ such that for all $z \in \mathbb{C}^{n} \backslash G_{r}$

$$
\frac{\left|\partial_{\mathbf{b}}^{p+1} F(z)\right|}{L^{p+1}(z)} \leq P_{3} \max \left\{\frac{\left|\partial_{\mathbf{b}}^{j} F(z)\right|}{L^{j}(z)}: 0 \leq j \leq p\right\} \text {. }
$$

If $z^{\prime} \in A:=H\left(g_{0}\right) \backslash \bigcup_{j=1}^{p}\left(G_{r}\left(g_{j}\right) \backslash H\left(g_{j}\right)\right)$, then there exists a sequence of points $z^{m} \in \mathbb{C}^{n} \backslash G_{r}$, satisfying (11) and such that $z^{m} \rightarrow z^{\prime}$ as $m \rightarrow \infty$. Substituting $z=z^{m}$ in (11) and passing to the limit as $m \rightarrow \infty$, we obtain that inequality is valid for all $z \in A \cup\left(\mathbb{C}^{n} \backslash G_{r}\right)$. Here we used joint continuity of the function $F$ for passing to the limit. If $\mathbb{C}^{n}=A \cup\left(\mathbb{C}^{n} \backslash G_{r}\right)$ (i.e., all zeros of the function $g^{\star}$ belong to $H\left(g^{\star}\right)$ ), then by Theorem 3 the function from the class $\mathcal{H}_{\mathbf{b}}^{n}$, obeying (9), has bounded L-index in the direction $\mathbf{b}$. Otherwise, $n\left(s, g^{\star}\right) \geq 1$.

Since $r \in\left(0, r^{\star}\right)$ and $r^{\star}=\sup _{s \geq 1} \frac{(s-1) \lambda_{1}(s)}{8\left(n\left(s, g^{\star}\right)+1\right)}$, there exists $r^{\prime} \geq 1$ such that $r \leq \frac{\left(r^{\prime}-1\right) \lambda_{1}\left(r^{\prime}\right)}{8\left(n\left(r^{\prime}, g^{\star}\right)+1\right)}$. Let $z^{0}$ be an arbitrary point from $\mathbb{C}^{n}$ and $K^{0}=\left\{z^{0}+t \mathbf{b}:|t| \leq r^{\prime} / L\left(z^{0}\right)\right\}$. Since the entire functions $g_{0}, g_{1}, \ldots, g_{p}, h$ have bounded $L$-index in the direction $\mathbf{b}$, by Theorem 2 the set $K^{0}$ contains at more $n\left(r^{\prime}, g^{\star}\right)$ zeros of these functions or $K^{0} \subset Z_{g^{*}}$. Let $c_{m}^{0}$ be zeros of the slice function $g^{\star}$ (that is $\left.g^{\star}\left(z^{0}+c_{m}^{0} \mathbf{b}\right)=0\right)$ such that $z^{0}+c_{m}^{0} \mathbf{b} \in K^{0} \cap\left(\left(Z_{h} \backslash\right.\right.$ $H(h)) \cup \bigcup_{j=0}^{p}\left(Z_{g_{j}} \backslash H\left(g_{j}\right)\right)$, where $m \in \mathbb{N}, m \leq n\left(r^{\prime}, g^{\star}\right)$. From $L \in Q_{\mathbf{b}}^{n}$ it follows that $L\left(z^{0}+c_{m}^{0} \mathbf{b}\right) \geq \lambda_{1}\left(r^{\prime}\right) L\left(z^{0}\right)$. Obviously,

$$
\begin{aligned}
\widetilde{K}_{m}^{0}:=\left\{z^{0}+t \mathbf{b}:\left|t-c_{m}^{0}\right| \leq \frac{r}{L\left(z^{0}+c_{m}^{0} \mathbf{b}\right)}\right\} & \subset\left\{z^{0}+t \mathbf{b}:\left|t-c_{m}^{0}\right| \leq \frac{\left(r^{\prime}-1\right) \lambda_{1}\left(r^{\prime}\right)}{8\left(n\left(r^{\prime}, g^{\star}\right)+1\right) L\left(z^{0}+c_{m}^{0} \mathbf{b}\right)}\right\} \\
& \subset K_{m}^{0}:=\left\{z^{0}+t \mathbf{b}:\left|t-c_{m}^{0}\right| \leq \frac{r^{\prime}-1}{8\left(n\left(r^{\prime}, g^{\star}\right)+1\right) L\left(z^{0}\right)}\right\} .
\end{aligned}
$$

Therefore, for $z^{0}+t \mathbf{b} \in K^{0} \backslash \bigcup_{z^{0}+c_{m}^{0} \mathbf{b} \in K^{0}} K_{m}^{0}(11)$ is true. Hence, for there points $z^{0}+t \mathbf{b}$ inequalities $L\left(z^{0}\right) \geq \frac{L\left(z^{0}+t \mathbf{b}\right)}{\lambda_{2}\left(r^{\prime}\right)}$ and (11) give us

$$
\begin{aligned}
\frac{\left|\partial_{\mathbf{b}}^{p+1} F\left(z^{0}+t \mathbf{b}\right)\right|}{L^{p+1}\left(z^{0}\right)} & \leq\left(\lambda_{2}\left(r^{\prime}\right)\right)^{p+1} \frac{\left|\partial_{\mathbf{b}}^{p+1} F\left(z^{0}+t \mathbf{b}\right)\right|}{L^{p+1}\left(z^{0}+t \mathbf{b}\right)} \\
& \leq P_{3}\left(\lambda_{2}\left(r^{\prime}\right)\right)^{p+1} \max _{0 \leq j \leq p}\left\{\frac{\left|\partial_{\mathbf{b}}^{j} F\left(z^{0}+t \mathbf{b}\right)\right|}{L^{j}\left(z^{0}+t \mathbf{b}\right)}\right\}
\end{aligned}
$$




$$
\begin{aligned}
& \leq P_{3}\left(\lambda_{2}\left(r^{\prime}\right)\right)^{p+1} \max _{0 \leq j \leq p}\left\{\frac{\left|\partial_{\mathbf{b}}^{j} F\left(z^{0}+t \mathbf{b}\right)\right|}{L^{j}\left(z^{0}\right)}\left(\frac{1}{\lambda_{1}\left(r^{\prime}\right)}\right)^{j}\right\} \\
& \leq P_{3}\left(\frac{\lambda_{2}\left(r^{\prime}\right)}{\lambda_{1}\left(r^{\prime}\right)}\right)^{p} \lambda_{2}\left(r^{\prime}\right) \max _{0 j \dot{0} p}\left\{\frac{\left|\partial_{\mathbf{b}}^{j} F\left(z^{0}+t \mathbf{b}\right)\right|}{L^{j}\left(z^{0}\right)}\right\}=P_{4} w_{z^{0}}(t),
\end{aligned}
$$

where $P_{4}=P_{3} \lambda_{2}\left(r^{\prime}\right)\left(\frac{\lambda_{2}\left(r^{\prime}\right)}{\lambda_{1}\left(r^{\prime}\right)}\right)^{p}$ and

$$
w_{z^{0}}(t)=\max \left\{\frac{\left|\partial_{\mathbf{b}}^{j} F\left(z^{0}+t \mathbf{b}\right)\right|}{L^{j}\left(z^{0}\right)}: 0 \leq j \leq p\right\} .
$$

Let $D$ be a sum of diameters $K_{m}^{0}$. Then $D \leq \frac{2\left(r^{\prime}-1\right) n\left(r^{\prime}, g^{*}\right)}{8\left(n\left(r^{\prime}, g^{*}\right)+1\right) L\left(z^{0}\right)}<\frac{r^{\prime}-1}{4 L\left(z^{0}\right)}$. Therefore there exist numbers $r_{1} \in\left[\frac{r^{\prime}}{4}, \frac{r^{\prime}}{2}\right]$ and $r_{2} \in\left[\frac{3 r^{\prime}+1}{4} ; r^{\prime}\right]$ such that for $z^{0}+t \mathbf{b} \in C_{j}=\left\{z^{0}+t \mathbf{b}:|t|=\frac{r_{j}}{L\left(z^{0}\right)}\right\}, j \in\{1,2\}$, one has $z^{0}+t \mathbf{b} \in K^{0} \backslash$ $\bigcup_{c_{m}^{0} \in K^{0}} K_{m}^{0}$. Choose arbitrary points $z^{0}+t_{1} \mathbf{b} \in C_{1}$ and $z^{0}+t_{2} \mathbf{b} \in C_{2}$ and connect them by a smooth curve $\gamma=\left\{z^{0}+t \mathbf{b}: t=t(s), 0 \leq s \leq 1\right\}$ such that $w_{z^{0}}(t) \neq 0$ and $\gamma \subset K^{0} \backslash \bigcup_{c_{m}^{0} \in K^{0}} K_{m}^{0}$.

In the following detailed description of construction of the curve we will use ideas from proof of Theorem 8 in [15] with adaptation for slice functions. For a construction of the curve $\gamma$ we connect $t_{1}$ and $t_{2}$ by a line $t(s)=\left(t_{2}-t_{1}\right) s+t_{1}, s \in[0,1]$. Let $t_{k}^{\star}$ be points on the line $t(s)$ such that $w_{z^{0}}\left(t_{k}^{\star}\right)=0$. The number of such points $m_{0}=m\left(z^{0}+t_{1} \mathbf{b}, z^{0}+t_{2} \mathbf{b}\right)$ is finite. Let $\left(t_{k}^{\star}\right)$ be a sequence of these points in ascending order of the value $\left|t_{1}-t_{k}^{\star}\right|, k \in\{1,2, \ldots, p\}$. We choose

$$
r_{0}<\min _{1 \leq k \leq m_{0}-1}\left\{\left|t_{k}^{\star}-t_{k+1}^{\star}\right|,\left|t_{1}^{\star}-t_{1}\right|,\left|t_{m_{0}}^{\star}-t_{2}\right|, \frac{r^{\prime}}{4 \pi L\left(z^{0}\right)}\right\} .
$$

Now we construct circles with centers at the points $t_{k}^{\star}$ and corresponding radii $r_{k}^{\prime}<\frac{r_{0}}{2^{k}}$ such that $w_{z^{0}}(t(s)) \neq 0$ for all $t$ on the circles. It is possible, because $F / \equiv 0$.

Every such circle is divided onto two semicircles by the line $t=t(s)$. The required piecewise-analytic curve consists with arcs of the constructed semicircles and segments of line $z_{1}^{*}(t)$, which connect the arcs in series between themselves or with the points $t_{1}, t_{2}$. If the curve intersects some set $K_{m}^{0}$ then we override the set by a semicircle with the center at the point $c_{m}^{0}$ and radius $\frac{r^{\prime}}{8\left(n\left(r^{\prime}, g^{*}\right)+1\right) L\left(z^{0}\right)}$.

The description shows that the curve can be chosen with the following estimate of its length

$$
|\gamma| \leq|\mathbf{b}|\left(\frac{\pi r_{1}}{L\left(z^{0}\right)}+\frac{r_{2}-r_{1}}{L\left(z^{0}\right)}+\frac{\pi r_{0}}{L\left(z^{0}\right)}+\frac{\pi n\left(r^{\prime}, g^{\star}\right) r^{\prime}}{8\left(n\left(r^{\prime}, g^{\star}\right)+1\right) L\left(z^{0}\right)}\right)<\frac{|\mathbf{b}|}{L\left(z^{0}\right)}\left(\frac{\pi r^{\prime}}{2}+r^{\prime}+\frac{\pi r^{\prime}}{8}\right)<\frac{3|\mathbf{b}| r^{\prime}}{L\left(z^{0}\right)} .
$$

Then on $\gamma$ inequality (12) is valid, that is

$$
\frac{\left|\partial_{\mathbf{b}}^{p+1} F\left(z^{0}+t(s) \mathbf{b}\right)\right|}{L^{p+1}\left(z^{0}\right)} \leq P_{4} w_{z^{0}}(t(s)), 0 \leq s \leq 1 .
$$

In view of the described construction, the function $z^{0}+t(s) \mathbf{b}$ is piece-wise analytic on $[0,1]$. Hence, for arbitrary $k \in \mathbb{Z}_{+}, j \in \mathbb{Z}_{+}, k \leq p$, either

$$
\frac{\left|\partial_{\mathbf{b}}^{k} F\left(z^{0}+t(s) \mathbf{b}\right)\right|}{L^{k}\left(z^{0}\right)} \equiv \frac{\left|\partial_{\mathbf{b}}^{j} F\left(z^{0}+t(s) \mathbf{b}\right)\right|}{L^{j}\left(z^{0}\right)},
$$

or the equality

$$
\frac{\left|\partial_{\mathbf{b}}^{k} F\left(z^{0}+t(s) \mathbf{b}\right)\right|}{L^{k}\left(z^{0}\right)}=\frac{\left|\partial_{\mathbf{b}}^{j} F\left(z^{0}+t(s) \mathbf{b}\right)\right|}{L^{j}\left(z^{0}\right)}
$$

holds only for a finite set of points $s_{k} \in[0 ; 1]$.

Then for the function $w_{z^{0}}(t(s))$ as maximum of such expressions $\frac{\left|\partial_{\mathbf{b}}^{j} F\left(z^{0}+t(s) \mathbf{b}\right)\right|}{L^{j}\left(z^{0}\right)}$ by all $j \leq p$ two cases are possible: 
1. In some interval of analyticity of the curve $\gamma$ the function $w_{z^{0}}(t(s))$ identically equals simultaneously to some derivatives, that is (13) holds. It means that $w_{z^{0}}(t(s)) \equiv \frac{\left|\partial_{\mathbf{b}}^{j} F\left(z^{0}+t(s) \mathbf{b}\right)\right|}{L^{j}\left(z^{0}\right)}$ for some $j \leq p$. Clearly, the function $\partial_{\mathbf{b}}^{j} F\left(z^{0}+t(s) \mathbf{b}\right)$ is analytic. Then $\left|\partial_{\mathbf{b}}^{j} F\left(z^{0}+t(s) \mathbf{b}\right)\right|$ is continuously differentiable function on the interval of analyticity except points where this directional derivative equals zero $\left|\partial_{\mathbf{b}}^{j} F\left(z^{0}+t(s) \mathbf{b}\right)\right|=0$. However, there are not the points, because in the opposite case $w_{z^{0}}(t(s))=0$. But it contradicts the construction of the curve $\gamma$.

2. In some interval of analyticity of the curve $\gamma$ the function $w_{z^{0}}(t(s))$ equals simultaneously to some derivatives at a finite number of points $s_{k}$, that is (14) holds. Then the points $s_{k}$ divide interval of analyticity onto a finite number of segments, in which of them $w_{z^{0}}(t(s))$ equals to one from the partial derivatives, i. e. $w_{z^{0}}(t(s)) \equiv \frac{\left|\partial_{\mathbf{b}}^{j} F\left(z^{0}+t(s) \mathbf{b}\right)\right|}{L^{j}\left(z^{0}\right)}$ for some $j \leq p$. As above, in each from these segments the functions $\left|\partial_{\mathbf{b}}^{j} F\left(z^{0}+t(s) \mathbf{b}\right)\right|$, and $w_{z^{0}}(t(s))$ are continuously differentiable except the points $s_{k}$.

Therefore, the function $g_{z^{0}}(t(s))$ is continuous on $[0,1]$ and continuously differentiable except, possibly, finite set of points. Moreover, for complex-valued function of real variable the following inequality $\frac{d}{d s}|\varphi(s)| \leq$ $\left|\frac{d}{d s} \varphi(s)\right|$ holds except the points $s$ where $\varphi(s)=0$. Therefore in view of (12) we obtain

$$
\begin{aligned}
\frac{d}{d s} g_{z^{0}}(t(s)) \leq \max _{0 \leq j \leq p}\left\{\frac{d}{d s} \frac{\left|\partial_{\mathbf{b}}^{j} F\left(z^{0}+t(s) \mathbf{b}\right)\right|}{\left.L^{j}\left(z^{0}\right)\right)}\right\} & \leq \max _{0 \leq j \leq p}\left\{\frac{\left|\partial_{\mathbf{b}}^{j+1} F\left(z^{0}+t(s) \mathbf{b}\right)\right|}{L^{j+1}\left(z^{0}\right)}\left|t^{\prime}(s)\right| L\left(z^{0}\right)\right\} \\
& \leq \max _{0 \leq j \leq p+1}\left\{\frac{\left|\partial_{\mathbf{b}}^{j} F\left(z^{0}+t(s) \mathbf{b}\right)\right|}{L^{j}\left(z^{0}\right)}\right\}\left|t^{\prime}(s)\right| L\left(z^{0}\right) \leq P_{5} g_{z^{0}}(t(s))\left|t^{\prime}(s)\right| L\left(z^{0}\right),
\end{aligned}
$$

where $P_{5}=\max \left\{1, P_{4}\right\}$. Integrating in variable $s$ and using (3), we establish

$$
\left|\ln \frac{g_{z^{0}}\left(t_{2}\right)}{g_{z^{0}}\left(t_{1}\right)}\right|=\left|\int_{0}^{1} \frac{1}{g_{z^{0}}(t(s))} \frac{d}{d s} g_{z^{0}}(t(s)) d s\right| \leq P_{5} L\left(z^{0}\right) \int_{0}^{1}\left|t^{\prime}(s)\right| d s \leq P_{5} L\left(z^{0}\right)|\gamma| \leq 3|\mathbf{b}| r^{\prime} P_{5},
$$

that is $g_{z^{0}}\left(t_{2}\right) \leq g_{z^{0}}\left(t_{1}\right) \exp \left\{3|\mathbf{b}| r^{\prime} P_{5}\right\}$. It is possible to choose $t_{2}$ such that

$$
\left|F\left(z^{0}+t_{2} \mathbf{b}\right)\right|=\max \left\{\left|F\left(z^{0}+t \mathbf{b}\right)\right|: z^{0}+t \mathbf{b} \in C_{2}\right\} .
$$

Hence,

$$
\max \left\{\left|F\left(z^{0}+t \mathbf{b}\right)\right|:|t|=\frac{3 r^{\prime}+1}{4 L\left(z^{0}\right)}\right\} \leq\left|F\left(z^{0}+t_{2} \mathbf{b}\right)\right| \leq g_{z^{0}}\left(t_{2}\right) \leq g_{z^{0}}\left(t_{1}\right) \exp \left\{3|\mathbf{b}| r^{\prime} P_{5}\right\} .
$$

Since $z^{0}+t_{1} \mathbf{b} \in C_{1}=\left\{z^{0}+t \mathbf{b}:|t|=\frac{r_{1}}{L\left(z^{0}\right)}\right\}$ and $r_{1} \in\left[\frac{r^{\prime}}{4}, \frac{r^{\prime}}{2}\right]$, for all $j \in\{1,2, \ldots, p\}$, by Cauchy's inequality in variable $t$ we obtain

$$
\frac{r^{\prime j}}{\left(4 L\left(z^{0}\right)\right)^{j}}\left|\partial_{\mathbf{b}}^{j} F\left(z^{0}+t_{1} \mathbf{b}\right)\right| \leq j ! \max \left\{\left|F\left(z^{0}+t \mathbf{b}\right)\right|:\left|t-t_{1}\right|=\frac{r^{\prime}}{4 L\left(z^{0}\right)}\right\} \leq p ! \max \left\{\left|F\left(z^{0}+t \mathbf{b}\right)\right|:|t|=\frac{3 r^{\prime}}{4 L\left(z^{0}\right)}\right\},
$$

i.e.

$$
g_{z^{0}}\left(t_{1}\right) \leq p ! \max \left\{1,\left(4 / r^{\prime}\right)^{p}\right\} \max \left\{\left|F\left(z^{0}+t \mathbf{b}\right)\right|:|t|=\frac{3 r^{\prime}}{4 L\left(z^{0}\right)}\right\} .
$$

From inequalities (15) and (16) it follows

$$
\max \left\{\left|F\left(z^{0}+t \mathbf{b}\right)\right|:|t|=\frac{3 r^{\prime}+1}{4 L\left(z^{0}\right)}\right\} \leq P_{6} \max \left\{\left|F\left(z^{0}+t \mathbf{b}\right)\right|:|t|=\frac{3 r^{\prime}}{4 L\left(z^{0}\right)}\right\},
$$

where $P_{6}=p ! \max \left\{1,\left(4 / r^{\prime}\right)^{p}\right\} \exp \left\{3|\mathbf{b}| r^{\prime} P_{5}\right\}$. Therefore, by Proposition 1 the function $F$ has bounded $L$-index in the direction $\mathbf{b}$. 


\section{References}

[1] Bandura A., Skaskiv 0., Slice holomorphic functions in several variables with bounded $L$-Index in direction, Axioms, 2019, 8(3), Article ID 88, DOI: 10.3390/axioms8030088

[2] Bandura A. I., Skaskiv O. B., Some criteria of boundedness of $L$-index in a direction for slice holomorphic functions of several complex variables, Ukr. Matem. Visn., 2019, 16(2), 154-180 (Engl. transl.: J. Math. Sci. (in print))

[3] Bandura A., Skaskiv 0., Analog of Hayman's theorem and its application to some system of linear partial differential equations, J. Math. Phys. Anal. Geom., 2019, 15(2), 170-191, DOI: 10.15407/mag15.02.170

[4] Bordulyak M. T., On the growth of entire solutions of linear differential equations, Mat. Stud., 2000, 13(2), 219-223

[5] Nuray F., Patterson R. F., Vector-valued bivariate entire functions of bounded index satisfying a system of differential equations, Mat. Stud., 2018, 49(1), 67-74, DOI: 10.15330/ms.49.1.67-74

[6] Kuzyk A. D., Sheremeta M. N., Entire functions of bounded l-distribution of values, Math. Notes, 1986, 39(1), 3-8, DOI: $10.1007 /$ BF01647624

[7] Kuzyk A. D., Sheremeta M. N., On entire functions, satisfying linear differential equations, Diff. Equations, 1990, 26(10), $1716-1722$

[8] Lepson B., Differential equations of infinite order, hyperdirichlet series and entire functions of bounded index, Proc. Sympos. Pure Math., 1968, 2, 298-307

[9] Macdonnell J. J., Some convergence theorems for Dirichlet-type series whose coefficients are entire functions of bounded index, Doctoral dissertation, Catholic University of America, Washington, USA, 1957

[10] Strelitz S., Asymptotic properties of entire transcendental solutions of algebraic differential equations, Contemp. Math., 1983, 25, 171-214, DOI: 10.1090/conm/025/730048

[11] Sheremeta M., Analytic functions of bounded index, Lviv, VNTL Publishers, 1999

[12] Bandura A., Skaskiv 0., Boundedness of the $L$-index in a direction of entire solutions of second order partial differential equation, Acta Comment. Univ. Tartu. Math., 2018, 22(2), 223-234, DOI: 10.12697/ACUTM.2018.22.18

[13] Bandura A., Skaskiv O., Filevych P., Properties of entire solutions of some linear PDE's, J. Appl. Math. Comput. Mech., 2017, 16(2), 17-28, DOI: 10.17512/jamcm.2017.2.02

[14] Bandura A. I., Skaskiv O. B., Entire functions of bounded $L$-index in direction, Mat. Stud., 2007, 27(1), 30-52 (in Ukrainian)

[15] Bandura A., New criteria of boundedness of $\mathbf{L}$-index in joint variables for entire functions, Math. Bull. Shevchenko Sci. Soc., 2016, 13, 58-67 (in Ukrainian) 